\begin{tabular}{ccc}
\hline & International Journal of Engineering \& Technology, $7(3.33)(2018) 139-142$ \\
SPC & International Journal of Engineering \& Technology \\
Website $:$ www.sciencepubco.com/index.php/IJET & Research paper \\
\hline
\end{tabular}

\title{
Warning System Using Multicast Transmission for Pedestrian and Pedal-Cyclist Safety Against the Electric-Powered Driving Means in CVCN Network
}

\author{
Bachyun Kim ${ }^{1}$, Yoseop Woo ${ }^{1}$, Iksoo Kim ${ }^{1 *}$ \\ ${ }^{1}$ Department of Information and Telecommunication Engineering of Incheon National University \\ 12-1 Songdo-dong Yeonsuku, Incheon, Korea \\ *Corresponding author E-mail:iskim@inu.ac.kr
}

\begin{abstract}
This paper deals with a warning system for the safety of pedestrians/pedal-cyclists against electric-powered driving means including hybrid/PHEV/EV/FCEV and electric wheel on minor roads. These roads are a subset of connected-vehicle communication network $(\mathrm{CVCN})$. The fatalities of pedestrians/pedal-cyclists declined recently compared to the early 2000s, but fatality rate of vehicle accidents is increasing. Clearly, this phenomenon will continue because of the increasing number of virtually silent hybrid/PHEV/EV/FCEV and electric wheels on the road.

The hybrid/PHEV/EV/FCEV such as green electric-powered ones that can reduce environmental pollution are much more dangerous than traditional vehicles to pedestrians/pedal-cyclists on minor roads. The main risk factor of the electric-powered vehicles is that they are very quiet on the road because of the use of electric motor instead of engine. Thus, the safety warning system that can notify pedestrians/pedal-cyclists the dangerous approaches of vehicles from their behind have to be provided on minor roads.

The proposed framework for safety warning system using multicast informs pedestrians/pedal-cyclists through smartphone when electric powered driving means are closing from their behind on minor roads. This is a new technology that uses vibration or sound of smartphone instead of artificial noise generation which is equipped to the electric powered driving means recently.
\end{abstract}

Keywords: Connected vehicle communication network(CVCN); Pedestrian Safety; Smart access agent; Electrical powered vehicle; RFID.

\section{Introduction}

The development of computing power of computer and wireless communication technology have contributed to the advancement of IOT(internet on thing) technology. This development has given a decisive effect on the fields of vehicle industry, intelligent traffic network, VANET(vehicle ad-hoc network), V2X(vehicle-toeverything) and ITS(intelligent transportation system[1, 2, 3].

The WHO's global status report on road safety 2015[4] has issued the number of casualties from traffic accidents(2013). It has reached more than 1.25 million per a year as on average 3,287 deaths a day, and about 50 million people has incurred serious or slightly injured each year due to vehicle crashes on the road all over the world[4, 5]. Especially, when we look at the causes of fatalities of the youth, those aged 15 to 29 , the number of death due to road traffic crashes outnumbers to that of disease. Injuries due to road traffic accidents impose a heavy economic burden on not only the nation but also their households. The fatalities of global traffic accidents have reached over 1.25 million in 2013 which is the highest record since 2001. Though the number of fatalities shows a slight decrease since 2014, profound researches are needed to reduce casualties from traffic accidents[4].

Thus, the various researches including autonomous driving system to reduce severe traffic accidents are proceeding during the last decade, and a main part of those researches is the fields of vehicle network such as vehicle communication network (VCN), vehicu- lar ad-hoc network (VANET) or V2X network. It is quite obvious these networks will be based on IOT technology[5, 6].

The CVCN(connected-vehicle communication network) network can provide multimedia service such as video and music data when driving on the road. These multimedia in CVCN network involve live-video of traffic accidents and road status monitoring information[7]. Multimedia data require vast network resources. The CVCN network in this paper uses multicast transmission not to deplete insufficient network resources in vehicle network. And the $\mathrm{CVCN}$ network involves the minor roads including parking lots.

The advancement of IOT technology in vehicle industry supports comfortable and safe driving including autonomous driving on the roadway. But the widespread use of the IOT will severely deplete the network resources. Thus, this paper deals with the safety driving system and seamless multimedia service mechanism to use network resources efficiently using multicast technique on the roads through the smart caching/switching/multicasting agent(SCSMA) in connected vehicle communication network(CVCN).

The rest of this paper continues as the following: Section 2 is analyzing statistical data about fatalities of pedestrians/pedal-cyclists and number of vehicle accidents. Section 3 describes the structure of warning system using multicast technique for the safety of pedestrians/pedal-cyclists and the efficient use of network resources in connected vehicle communication network $(\mathrm{CVCN})$. Section 4 deals with the procedures that are composed of 3-parts using multicast transmission. Finally, we represent the conclusion about the 
proposed warning system for the safety of pedestrians and pedalcyclists.

\section{The Statistical Data of Pedestrian Fatalities of Vehicle Collisions on the Road}

The Mail Online published in March 2015[8] states that green Hybrid/PHEV/EV vehicles are much more dangerous than traditional vehicles that use fossil fuels as gasoline and diesel on minor roads to pedestrians and pedal-cyclists. But those vehicles can reduce environmental pollution. So they are expected to increase significantly for next 10 years. Other reasons of the increase are depletion of fossil fuels and the tax support of most governments. The subsidy of electric-powered vehicles is almost more than $\$ 10,000$ all over the world, though it varies by car models[9].

The number of injuries and fatalities due to the vehicle accidents may increase fast because of they have small running noise. Since almost all of the people regardless of age use smartphones in general while they are walking, the number of casualties can increase on minor roads.

The Fig. 1 shows the number of vehicle accidents and fatalities by vehicle from 2005 to 2015 in OECD 31-countries and 33countries, respectively[10, 11].

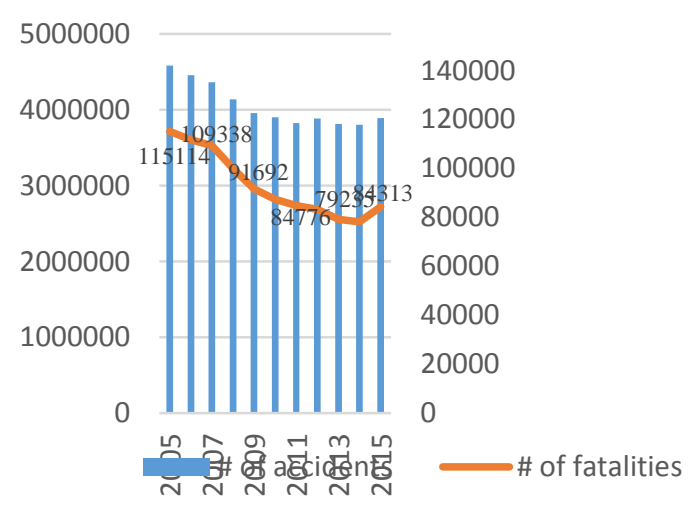

Fig. 1: The number of vehicle accidents and fatalities in OECD countries from 2005 to 2015

As shown in Fig. 1, the number of vehicle accidents and fatalities except 2015 is decreasing. This is the result of road traffic safety effort of each OECD country. But recently, the supply expansion of smartphones through the entire age group except early children and the amazing growth in the use of them on the road can lead to serious vehicle accidents. The wide spread of electric-powered driving means including electric-wheels for the next ten years can be the cause of increasing the vehicle accidents. This may give an important influence especially on minor roads because there is little noise.

Fig. 2 shows the number of fatalities(bar graph) and injuries(band graph) of pedestrians from 2005 to 2017 in Korea. The types of pedestrian accidents caused by vehicle are crossing the crosswalk, crossing the street, roadside zone in passage, walking down the sidewalk and others.

As shown in Fig. 2, we know that the number of fatalities and injuries by vehicle in Korea tend to decline.

Fig. 3 shows the number of pedal-cyclists' fatalities(bar graph) and injuries(band graph) from 2005 to 2017 in Korea. As shown in Fig. 3, we know that the number of fatalities and injuries of pedal-cyclists hit the peak by 2009 and 2015, respectively and then decrease later[10].

Through Fig. 1 to Fig. 3, the blue bar indicates the number of vehicle accidents, fatalities of pedestrians and that of pedal-cyclists, respectively. All the blue bars represent the left-handed y-axis. Orange band graphs indicate the number of fatalities by vehicle, injuries of pedestrians and pedal-cyclists, respectively. All the orange bars represent the right-handed y-axis.

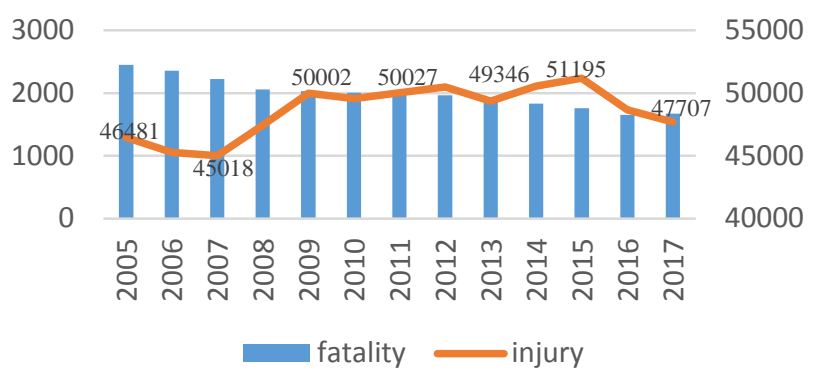

Fig. 2: The number of pedestrian casualties in Korea from 2005 to 2017

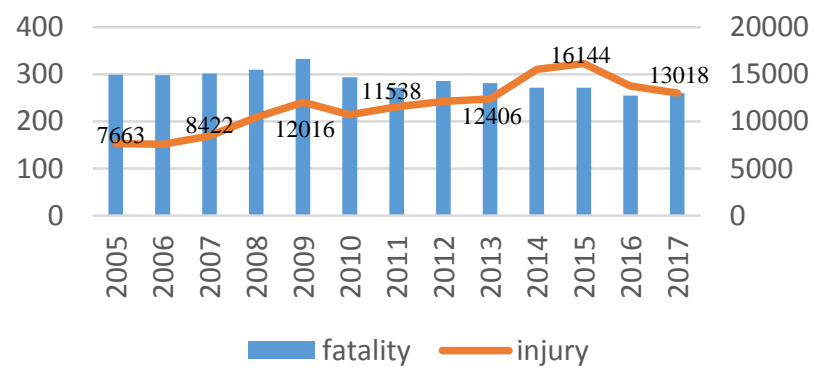

Fig. 3: The number of pedal-cyclist casualties in Korea over 2005 to 2017

Through Fig.1 to Fig. 3, these results may change in the case that many virtually silent electric powered vehicles will be driven on the road and more interesting services will be supported on various mobile devices such as smartphone. Thus, the researches that can provide guaranteed traffic safety on the road against all types of electric powered driving means including Hybrid/PHEV/EV and FCEV have to be proceeded.

\section{The Structure of Warning System Using Multicast Transmission for the Safety of Pedestrian/Pedal-Cyclists on Minor Roads}

The connectivity in CVCN (connected vehicle communication network) network covers infotainments, safe system for all kinds of vehicles and wireless nomadic devices that use communication infrastructures [11, 12]. Thus, CVCN network may include autonomous driving system which can be the root of the fourth industrial revolution.

The proposed warning system using multicast transmission strategy informs pedestrians and pedal-cyclists through the smartphone when all types of electric powered driving means including $\mathrm{Hy}$ $\mathrm{brid} / \mathrm{PHEV} / \mathrm{EV} / \mathrm{FCEV}$ vehicles and electric-powered wheels as well as conventional gasoline(diesel)-driven vehicles are closing from their behind on minor roads that forms the CVCN network. Thus the proposed warning system can enable pedestrians to have comfortable walking and leisure time safely.

The structure of the proposed warning system is composed of a server that supports safe system and infotainments, some smart caching/switching/multicasting agents(SCSMA), smartphones of pedestrians and RFID tag equipped in the electric powered driving means. The smart caching/switching/multicasting agents(SCSMA) perform multicast grouping to group some pedestrians that enter minor roads during pre-defined time, generate the multicast ad- 
dress and send it to the pedestrians' and pedal-cyclists smartphones. They(SCSMA) assign holding-time to multicast group according to the length of minor road and discards multicast group(multicast group address) when the allotted holding-time is passed.

The pedestrians' and pedal-cyclists' smartphone receives multicast group address from the SCSMA, and then joins its multicast group when they enter the minor road. Then they leave the multicast group when they get out of the minor road or when the allotted holding-time to their multicast group is passed.

The electric powered driving means such as electric-powered wheels and vehicles access SCSMA when they enter the minor road. The SCSMA sends command to activate their RFID tag when it sent the multicast group address to the pedestrians' and pedal-cyclists' smartphone. The electric powered driving means never activate their RFID tag when pedestrian and pedal cyclist do not exist on the minor road.

\section{The Operation Mechanism of the Safety System for Pedestrian/Pedal-Cyclist Against Electric-Powered Driving Means on Minor Roads}

The operation procedure of the proposed safe warning system for pedestrians and pedal-cyclists in the minor road is composed of three parts: the procedures for the smart caching/switching/multicasting agents(SCSMA), vehicles and pedestrians'/pedal-cyclists's smartphones.

The SCSMAs have the function of performing multicast transmission strategy and caching technology for multimedia as well as general function of common access point. Thus, SCSMAs have the function of multicast grouping, joining and leaving the multicast group including caching.

All the entering vehicles on minor roads have a mobile device that can join and leave the multicast group.

\subsection{The Procedure for Smart Cach- ing/Switching/Multicasting Agents (SCSMA) on Minor Roads}

i) As pedestrians, pedal-cyclists and vehicles enter into the minor road, the SCSMA(smart caching/switching/multicast- ing agents: SCSMA_\#xxxx)that locate the entering point generates a specific multicast group address(Mcast_\#y) and vehicle ID(equipped RFID tag: vehicle_\#k), respectively.

ii) The SCSMA_\#xxxx performs multicast grouping during a predefined multicast grouping time. The SCSMA_\#xxxx assigns the same multicast group address to the pedestrians and pedal-cyclists entering into the minor road within a specific grouping time

iii) The SCSMA(SCSMA_\#xxxx) notifies the adjacent two SCSMAs(SCSMA_\#xxxx+1 and SCSMA_\#xxxx-1) pedestrian's multicast group address(assigned in step ii) and the vehicle ID (vehicle_\#k)

iv) The SCSMAs(SCSMA_\#xxxx, \#xxxx+1 and \#xxxx-1) initializes their timer within a specific multicast grouping time for pedestrians, pedal-cyclists and vehicle_k when they try to join the multicast group address(Mcast_\#y)

v) The SCSMA_\#xxxx notifies the smartphone of pedestrians and pedal-cyclists to launch safety application program(APs) to cope with the closing of vehicle_k

vi) The SCSMA_\#xxxx sends a warning signal to pedestrians and pedal-cyclists with a specific multicast group address(Mcast_\#y)

vii) The SCSMA_\#xxxx notifies SCSMA_\#xxx+1 or SCSMA_\#xxxx-1 to send a warning signal to the multicast group
address(Mcast_\#y) according to the direction of forwarding pedestrians and pedal-cyclists

viii) The SCSMA_\#xxxx stops sending the warning signal to the multicast group address(Mcast_\#y) when the prescribed time has passed.

ix) The SCSMA(SCSMA_\#xxxx-1 or SCSMA_\#xxxx+1) sends the warning signal to the multicast group address(Mcast_\#y) for the prescribed time when the pedestrians and pedal-cyclists try to join multicast group address(Mcast_\#y)

x) The SCSMA(SCSMA_\#xxxx-1 or SCSMA_\#xxxx+1) sends the adjacent SCSMAs(SCSMA_\#xxxx-2 or SCSMA_\#xxxx+2) with the pedestrian's multicast group address(Mcast_\#y) and the vehicle ID(vehicle_\#k)

xi) The last SCSMA(SCSMA_\#xxxx+z) checks the multicast group(Mcast_\#y) or the vehicle(vehicle_\#k) whether they passed out through the minor road or not according to the prescribed time.

xii) The last SCSMA(SCSMA_\#xxxx+z) sends the passage signal to the SCSMA(SCSMA_\#xxxx) when the multicast group(Mcast_\#y) time is over or the vehicle(vehicle_\#k) has passed out the minor road

xiii) The starting SCSMA_\#000 initializes the multicast group(Mcast_\#y) provided to pedestrians/pedal cyclists or vehicles(vehicle_\#k) that have passed out the minor road

\subsection{The Procedure for Entering Electric-Powered Vehi- cles to the Minor Road}

i) The electric-powered vehicle access the nearest SCSMA (SCSMA_\#xxxx) when it enters the minor road.

ii) The electric-powered vehicle launches its $\mathrm{AP}$ (application program)

iii) The vehicle acquires vehicle ID(vehicle_\#k) from the SCSMA_\#xxxx

iv) The vehicle_\#k initializes the equipped RFID tag working after sending the acknowledged signal to the accessed SCSMA( SCSMA_\#xxxx)

v) The vehicle_\#k receives the prescribed equipped RFID tag working time from the SCSMA_\#xxxx.

vi) The vehicle_\#k stops the RFID tag from working when the prescribed time has passed or when it receives the signal of complete passage from the last SCSMA(SCSMA_\#xxxx+z) on the minor road or when it receives signal that the assigned multicast groups are not on the minor road.

vii) If there are other multicast groups ahead of the vehicle_\#k on the minor road, then goto step iv)

\subsection{The Procedure for the Pedestrian and Pedal- Cyclists}

i) The pedestrian and pedal-cyclists access the nearest SCSMA(SCSMA_\#xxxx) as soon as they enter the minor road

ii) The pedestrian and pedal-cyclists acquire a specific IP address from the SCSMA_\#xxxx

iii) The pedestrian and pedal-cyclists launch the safety application program(APs) of their smartphones.

iv) The application program(APs) of pedestrian's and pedalcyclist's smartphone receives the multicast group address(Mcast_\#y) from the SCSMA_\#xxxx.

v) The application program(APs) of pedestrian's and pedalcyclist's smartphone joins the Mcast_\#y group. 
vi) If it receives warning signal through the multicast group address(Mcast_\#y), the application program(APs) vibrates.

Else then, it activates without vibration

vii) If they are on the minor road when the prescribed time has passed, it acquires a new specific IP address from the SCSMA_\#xxxx or other SCSMA_\#kkkk according to its location on the minor road

viii) The application program(APs) leaves the multicast group (Mcast_\#y) when the prescribed time has passed or when they are not on the minor road and stops sending the warning signal

The step viii) of the procedure for the pedestrian and pedalcyclists is the case that they are still on the minor road when the prescribed time has passed, that is when other vehicles are on that road.

\section{Conclusion}

The safety of pedestrians and pedal-cyclists is a matter of utmost importance while the hybrid vehicle/PHEV/EV and FCEV vehicles are driving on the roads. Besides, the electric-powered pedalcyclists including electric wheel for leisure may become an assailant or a victim on the road. These electric-powered driving means have little noise during driving on the roads. This phenomenon leads to improvement of the urban environment but it may be a danger to pedestrians and pedal-cyclists on the minor roads including parking lots.

The framework of the warning system proposed in this paper can provide the safety of pedestrians and pedal-cyclists against little noised vehicles approaching from behind on the minor roads that composes the CVCN network. This warning system gives support in the type of an alarm, vibration or text on the smartphone with the aid of SCSMA on the minor road. The proposed technology adapts multicast mechanism in order to use the network resources effectively. This warning system may not deplete the network resources on the existing services because it uses multicast transmission. The research for the implementation of warning system with multicast through the smartphone is in progress and it can contribute significantly to the safety of pedestrians and pedalcyclists until the fully-autonomous driving vehicles are driving on the roads.

\section{Acknowledgement}

This work was supported by the Incheon National University Research Grant in 2016

\section{References}

[1] M. Hawes, "Connected and Autonomous Vehicle-The UK Economic Opportunity", https://www.kpmg.com/BR/en/Estudos_Analises/artigosepublicaco es/Documents/Industrias/ConnectedAutonomous-VehiclesStudy.pdf, March (2015)

[2] L'Atelier - Paris, “\#CES2016: Smart Home and Smart Car's ecosystems are converging", http://www.atelier.net/en/trends/articles/ces2016-smart-home-andsmart-ecosystems-areconverging_439914, Febuary (2016)

[3] A Awer, A. Feese and A. Lockwood. "History of intelligent transportation system", May (2016) https://www.its.dot.gov/history/index.html.

[4] GLOBAL STATUS REPORT ON ROAD SAFETY 2015, published in WHO (2015)http://www.who.int/violence_injury_prevention/road_safety_ status/2015/en/http://apps.who.int/iris/bitstream/10665/189242/1/9789 241565066_eng.pdf?ua=1

[5] 5G Americas White Paper, "Cellular V2X Communications Towards
(2018)http://www.5gamericas.org/files/9615/2096/4441/2018_5G_ Ameri-

cas_White_Paper_Cellular_V2X_Communications_Towards_5G Final_for_Distribution.pdf

[6] Wai Chen, "Vehicular Communications and Networks, Architectures, Protocols, Operation and Deployment", Woodhead Publishing (2015)

[7] Iksoo Kim and Yosup Woo, "The multicast system for seamless live multimedia in WLAN", EURASIP JWCN, No. 246 (2013)

[8] Daily Mail Online March (2015), http://www.dailymail.co.uk/sciencetech/article-3011957/ Nearlysilent-electric-hybrid-cars-risk-pedestrians-Walkers-40-likelyinvolved-accident.html

[9] Ministry of Environment of Korea, "Eco-friendly Vehicle", Dec. (2015)

http://www.me.go.kr/home/web/board/read.do?menuId=10181\&org $\mathrm{Cd}=\&$ boardId $=589780$ \&boardMasterId $=54$

[10] International Transport Forum, "Pedestrian Safety, Urban Space and Health", https://read.oecd-ilibrary.org/transport/pedestriansafety-urban-space-and-health_9789282103654-en\#page41

[11] Traffic accidemt analysis system, the Korea Road Traffic Authority (2017)http://taas.koroad.or.kr/sta/acs/exs/typical.do?menuId=WEB KMP OVT UAS PDS\#

[12] Iksoo Kim and Yosup Woo, "Alarm System for Pedestrians Safety Against Hybrid/PHEV/EV Vehicle on Minor Road", International Journal of u- and e- Service, Science and Technology, vol. 9, No. 7(2016) 361-368 